\title{
Frequency of Hypomagnesaemia following Total and Near-total Thyroidectomy and its relationship with Hypocalcaemia
}

\author{
Sana Viqar ${ }^{1}$, Tasleem Akhtar ${ }^{2}$, Nadeem Ikram³ and Naeem Zia ${ }^{4}$ \\ ${ }^{1}$ Rawalpindi Medical University, Rawalpindi, Pakistan \\ ${ }^{2}$ Department of Vascular Surgery, Russells Hall Hospital, Dudley, UK \\ ${ }^{3}$ Department of Pathology, Benazir Bhutto Hospital, Rawalpindi, Pakistan \\ ${ }^{4}$ Department of Surgery Unit 1, Benazir Bhutto Hospital, Rawalpindi, Pakistan
}

\begin{abstract}
Objective: To determine the frequency of hypomagnesaemia in patients undergoing thyroidectomy and evaluate its relationship with postoperative hypocalcaemia.

Study Design: Observational, cross-sectional study.

Place and Duration of Study: Surgical Unit 1, Benazir Bhutto Hospital, Rawalpindi, Pakistan from April 2017 to May 2019.

Methodology: Preoperative and 24-hour postoperative samples for serum calcium and magnesium of 75 patients undergoing total and near-total thyroidectomy were taken. Postoperative signs and symptoms of hypocalcaemia were documented. Data was analysed on SPSS version 22, using independent and paired t-test, Pearson correlation, linear regression, Chi-square test and Fisher's Exact test.

Results: Postoperatively 49 (65.3\%) patients developed hypocalcaemia and 8 (10.7\%) developed hypomagnesaemia. Postoperative hypocalcaemia was significantly associated with hypomagnesaemia $(p=0.03)$ with a significant positive linear correlation. Ten patients (13.3\%) developed symptomatic hypocalcaemia; however, it was not associated with postoperative hypomagnesaemia. Postoperative hypocalcaemia and hypomagnesaemia were significantly associated with preoperative calcium level $(p=$ 0.03 and 0.04 , respectively). There was significantly lower calcium level in patients with hypomagnesaemia $(p=0.002)$ and a significant fall in magnesium level in patients developing hypocalcaemia $(p=0.044)$.

Conclusion: Postoperatively hypocalcaemia was prevalent following thyroidectomy, while hypomagnesaemia was not common. Hypomagnesaemia and a fall in magnesium level after surgery was associated with hypocalcaemia; but not with symptomatic hypocalcaemia. Monitoring of magnesium levels in severe or persistent hypocalcaemia is recommended. While this study proves a relationship between calcium and magnesium, its clinical implication must be further studied.
\end{abstract}

Key Words: Calcium, Magnesium, Postoperative, Hypocalcaemia, Hypomagnesaemia, Thyroidectomy.

How to cite this article: Viqar S, Akhtar T, Ikram N, Zia N. Frequency of Hypomagnesaemia following Total and Near-total Thyroidectomy and its relationship with Hypocalcaemia. J Coll Physicians Surg Pak 2021; 31(05):571-575.

\section{INTRODUCTION}

A biochemical relationship exists between magnesium and parathyroid hormone (PTH). Low serum magnesium stimulates PTH secretion. Very low magnesium has a paradoxical effect on PTH secretion by mimicking the activation of the calcium sensing receptor causing inhibition of PTH secretion. ${ }^{1,2}$

Correspondence to: Dr. Sana Viqar, Rawalpindi Medical University, Benazir Bhutto Hospital, Rawalpindi, Pakistan E-mail: sana.viqar27@gmail.com

Received: July 17, 2020; Revised: October 12, 2020;

Accepted: December 01, 2020

DOI: https://doi.org/10.29271/jcpsp.2021.05.571
Another relationship lies in the PTH-mediated renal absorption of magnesium. ${ }^{3}$ In presence of low levels of parathyroid hormone, there is low serum magnesium. This, in turn, decreases PTH secretion and further causes hypocalcaemia. It has been revealed that with a fall in magnesium below certain level, PTH resistance on organs also develops. ${ }^{4}$ One experiment showed that patients when given magnesium-deficient diet, showed a decrease in calcium level. ${ }^{5}$

If this biochemical relationship exists, itmust have clinical implications, particularly in patients undergoing thyroidectomy in whom transient and permanent hypocalcemia and hypoparathyroidism has been reported. ${ }^{6,7}$ Transient hypocalcaemia is a common outcome of thyroidectomy, reported in $45 \%$ patients. ${ }^{8,9}$ Numerous studies have been conducted to assess hypocalcaemia in patients after total thyroidectomy. Likewise, hypomagnesaemia has been noted after parathyroid surgery. ${ }^{10}$ 
Yet, there is limited study on the role of hypomagnesaemia post-thyroidectomy. In literature, a few studies have been conducted to study the role of magnesium in relation to the development of hypocalcaemia after thyroidectomy., ${ }^{9,11-14}$

Many studies have shown a significant association between hypomagnesaemia and hypocalcemia, ${ }^{11-13}$ while some have not. ${ }^{14,15}$ If the relationship of postoperative hypocalcaemia and hypomagnesaemia, ${ }^{11-13}$ can be established then therapeutic role of magnesium can be considered to treat hypocalcaemia, and correct the chain of pathways, leading to hypoparathyroidism following thyroidectomy. The purpose of this study was to find the frequency of hypomagnesaemia following thyroidectomy, and evaluate its association with postoperative hypocalcaemia.

\section{METHODOLOGY}

An observational cross-sectional study was conducted in Surgical Unit 1, Benazir Bhutto Hospital, Rawalpindi from April 2017 to May 2019 with the approval from the Ethical Review Committee of Rawalpindi Medical University. Total sample size was 75 patients with non-randomised consecutive sampling technique. All patients undergoing total or near-total thyroidectomy were included in the study. Those undergoing any other thyroidectomy or having any known parathyroid disease were excluded. Patients fulfilling the criteria were selected and preoperatively blood samples were taken for serum calcium and magnesium without use of a tourniquet. All information was filled on self-produced proformas. Informed consent was taken from the patients, who were prepared for surgery as per the department's protocol. Thyroidectomy was performed using extra capsular dissection. Peroperative intra-venous fluids used were documented. Samples for serum calcium and magnesium were taken 24-hour after surgery and signs and symptoms of hypocalcaemia noted. Signs included Chvostek's and Trousseau's signs. Symptoms included numbness, tingling sensation in extremities or face and muscle cramps or spasm. Symptomatic patients were managed with slow intravenous calcium gluconate. They were discharged when stable and showing no signs or symptoms of hypocalcaemia.

Hypocalcaemia was defined as serum calcium $<8.5 \mathrm{mg} / \mathrm{dl}$. Hypomagnesaemia was defined as serum magnesium $<1.9 \mathrm{mg} / \mathrm{dl}$. Symptomatic hypocalcaemia was defined as presence of symptoms or signs of hypocalcaemia. All blood tests were performed at Bio-path Lab. Data was entered and analysed on SPSS version 22. Qualitative data was expressed as numbers and percentages, while quantitativeas mean with standard deviation. Analytical tests including independent and paired t-test, Pearson correlation, linear regression, Chi-square test and Fisher's Exact test were applied with a significant association at $p$ value less than 0.05 .

\section{RESULTS}

Out of 75 patients, 69 (92\%) were females. Mean age was $38.6 \pm$ 12.08 years. Forty-two (56\%) patients were less than 40 years.
Patients with hyperthyroidism symptoms were 19 (25.3\%) and those with pressure symptoms were $20(26.7 \%)$. Out of total patients, $56(74.7 \%)$ underwent total and $16(21.3 \%)$ underwent near-total thyroidectomy.

Preoperatively, 53 (70.7\%) patients were normocalcemic, while $16(21.3 \%)$ were hypocalcemic. Mean calcium level was $8.77 \pm$ $0.53 \mathrm{mg} / \mathrm{dl}$ (range 7.4-10.10 mg/dl). All patients were normomagnesemic preoperatively. Mean magnesium level was $2.2 \pm$ $0.19 \mathrm{mg} / \mathrm{dl}$ (range 1.9-2.8 mg/dl). There was a significant positive linear correlation between preoperative calcium and magnesium levels $(r=0.401, p=0.001)$ with $R^{2}=0.161$ as shown in Figure 1.

Postoperatively 49 (65.3\%) patients developed hypocalcaemia. Mean calcium level was $8.2 \pm 0.63 \mathrm{mg} / \mathrm{dl}$ (range $7-9.6 \mathrm{mg} / \mathrm{dl}$ ). Eight (10.7\%) patients developed post-operative hypomagnesaemia. Mean magnesium level was $2.08 \pm 0.23$ $\mathrm{mg} / \mathrm{dl}$ (range 1.2-2.5 mg/dl). Eight patients developing hypomagnesaemia were all found to have hypocalcaemia. All patients with normal calcium levels had normal magnesium levels. This relationship between frequency of patients developing postoperative hypocalcemia and hypomagnesemia is shown in Figure 2. Postoperatively hypocalcemia was significantly associated with hypomagnesemia ( $p$ value $=0.03$ ) with a positive linear correlation $(r=0.556, p<0.001)$ and $R^{2}=0.31$ as shown in Figure 3 . The mean drop in calcium and magnesium levels after surgery was $0.499 \pm 0.6$ and $0.12 \pm 0.27$, respectively. Paired t-test showed that the difference between pre-and postoperative levels for calcium and magnesium was significant ( $p<0.001$ and $p=0.001$, respectively). Fall in calcium was significantly correlated to fall in magnesium ( $p$ $<0.001$ ) with positive linear relation $\left(R^{2}=0.18\right)$.

Mean postoperative calcium levels in normomagnesemic group was $8.30 \pm 0.61 \mathrm{mg} / \mathrm{dl}$ and hypomagnesemic group was $7.6 \pm 0.31 \mathrm{mg} / \mathrm{dl}$; using Independent T test, this was a significant difference $(p=0.002)$. Linear regression revealed that with every $1 \mathrm{mg} / \mathrm{dl}$ increase in magnesium, calcium increased by $1.485 \mathrm{mg} / \mathrm{dl}$; this was statistically significant with $p<0.001$ (Equation: calcium $=1.485$ (magnesium) +5.126$)$. Furthermore, those developing hypocalcemia showed a greater mean fall in magnesium levels after surgery than did those with normal postoperative calcium $(0.17 \pm 0.29$ vs. $0.03 \pm 0.21$, $\mathrm{p}=0.044$ ).

Ten patients (13.7\%) developed symptomatic hypocalcemia. Out of those developing hypocalcemia, $9 / 47(19.1 \%)$ had postoperative hypocalcemic symptoms ( $p=0.149, O R=5.7$ ) and only $1 / 8(12.5 \%)$ patient with hypomagnesemia developed symptoms. There was no significant association of symptomatic hypocalcemia with postoperative hypocalcemia $(p=$ 0.149 ) or hypomagnesemia ( $p>0.999$ ). Postoperative hypocalcemia and hypomagnesemia was significantly associated with preoperative calcium level ( $p=0.03$, OR: 5.0 and $p=0.04$, respectively). A summary of common distribution of variables is given in Table I. 
Table I: Distribution of common variables.

\begin{tabular}{|c|c|c|c|c|c|}
\hline & & \multicolumn{2}{|c|}{ Postoperative hypocalcemia } & \multicolumn{2}{|c|}{ Postoperative hypomagnesemia } \\
\hline & & Frequency & p-value & Frequency & p value \\
\hline Age category & $>40$ years & $24 / 31(77.4 \%)$ & 0.06 & $3 / 30(10 \%)$ & $>0.999$ \\
\hline Gender & Female & $47 / 68(69.1 \%)$ & 0.171 & $8 / 65(12.3 \%)$ & 0.41 \\
\hline Retrosternal goiter & Yes & $6 / 7(85.7 \%)$ & 0.242 & $0 / 6(0 \%)$ & $>0.999$ \\
\hline \multirow{2}{*}{ Surgery } & Total thyroidectomy & $37 / 54(69 \%)$ & \multirow{2}{*}{0.81} & $6 / 49(12.2 \%)$ & \multirow{2}{*}{0.75} \\
\hline & Near-total thyroidectomy & $11 / 16(69 \%)$ & & $1 / 16(6.3 \%)$ & \\
\hline \multirow{2}{*}{ Symptoms } & Hyperthyroidism & $16 / 19(84 \%)$ & \multirow{2}{*}{0.843} & $4 / 18(22.2 \%)$ & \multirow{2}{*}{0.68} \\
\hline & Pressure symptoms & $15 / 20(79 \%)$ & & $1 / 17(6 \%)$ & \\
\hline Preoperative calcium & Hypocalcemia & $13 / 1586.7 \%)$ & 0.03 & $4 / 15(26.7 \%)$ & 0.04 \\
\hline \multirow[t]{2}{*}{ Histology } & Benign & $29 / 43(67.4 \%)$ & \multirow{2}{*}{0.65} & $6 / 41(14.6 \%)$ & \multirow{2}{*}{$>0.999$} \\
\hline & Malignant & $5 / 6(83.3 \%)$ & & $0 / 5(0 \%)$ & \\
\hline Symptoms of hypocalcemia & Present & $9 / 10(90 \%)$ & 0.149 & $1 / 10(10 \%)$ & $>0.999$ \\
\hline
\end{tabular}

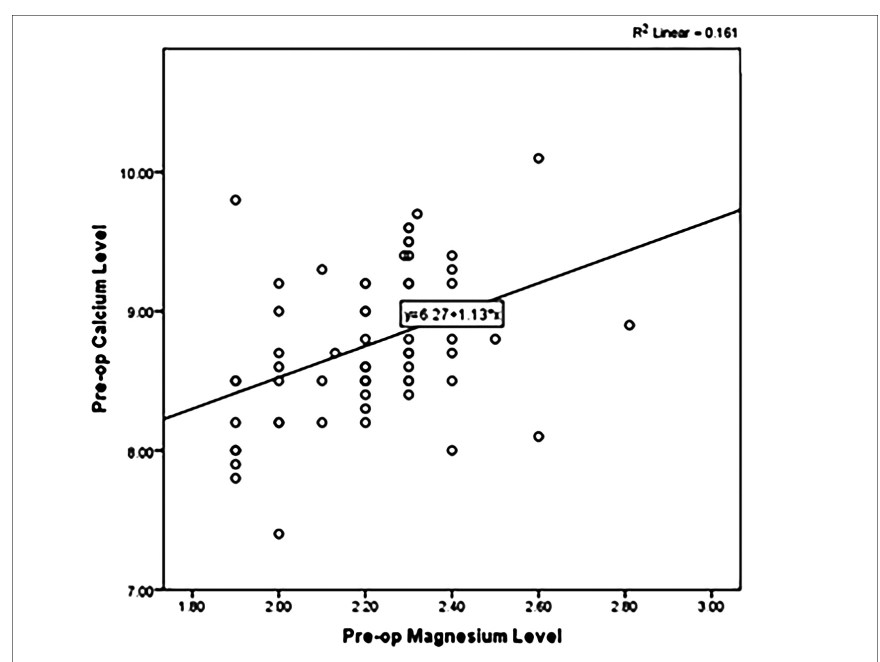

Figure 1: Preoperative $\mathrm{Ca}$ and Mg correlation.

\section{Post-operative levels of Calcium and Magnesium}

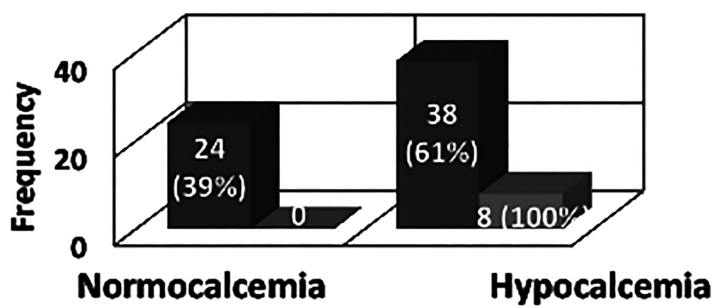

- Normomagnesemia $\square$ Hypomagnesemia

Figure 2: Relation between postoperative hypocalcemia and hypomagnesemia.

\section{DISCUSSION}

In this study, preoperatively no patient was hypomagnesemic, 16 patients $(21.3 \%)$ were hypocalcemic and none were symptomatic. In the study by Wilson et al., only one patient had hypocalcemia preoperatively. ${ }^{12}$ Cherian et al. showed pre-op hypocalcemia in $5(10 \%)$ and hypomagnesemia in $12(24 \%)$ patients. ${ }^{11}$ Postoperatively hypocalcemia was seen in $49(66 \%)$ of the patients as compared to other studies, where it was seen in $30-68 \%$ of patients ${ }^{9,11-13,16}$ and specifically in Pakistan reported in $13-50 \%$ patients. ${ }^{17-19}$ Only
$8(10.7 \%)$ patients developed hypomagnesemia in this study as compared to $13-70 \%$ in other studies. ${ }^{11,13,16}$ Selection of different definitions of hypocalcemia and hypomagnesemia, any unknown comorbidity influencing electrolyte imbalance or different surgical techniques can account for differences in frequencies. Variation in timing of preoperative sampling and delivery of blood samples to the laboratory was noted in some of the patients. Ono et al. demonstrated that blood calcium falls when blood sample is stored more than 24 hours. ${ }^{20}$

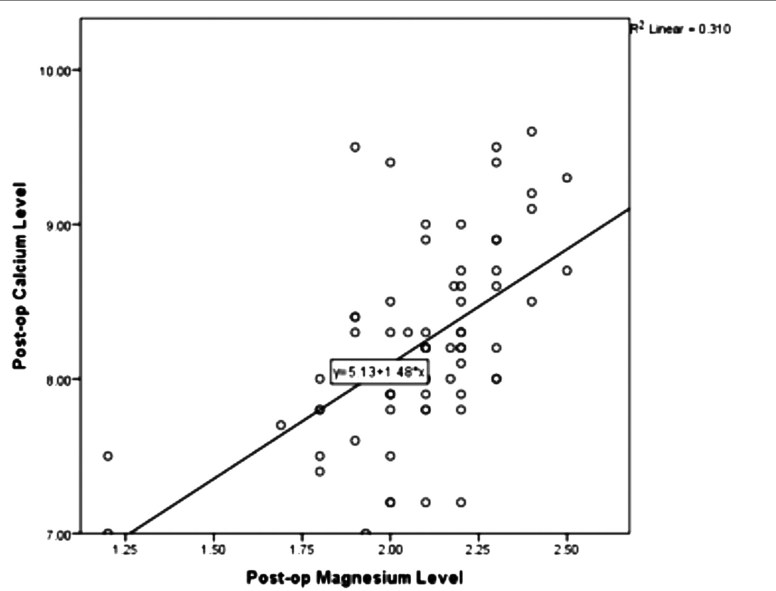

Figure 3: Postoperative $\mathrm{Ca}$ and $\mathrm{Mg}$ correlation.

There was a significant association between postoperative hypocalcemia and hypomagnesemia, similar to findings of Garrhy et al. ${ }^{13}$ All eight patients with hypomagnesemia were hypocalcemic. Significantly lower mean calcium level in patients developing hypomagnesemia than those with normomagnesemia was in coherence to the findings of study by Wang et al. ${ }^{21}$ Similar to this study, others have shown a significant positive correlation between calcium and magnesium levels. ${ }^{12,13}$

In this study, 10 (13.3\%) patients developed signs and symptoms of hypocalcemia in contrast to other studies, where it was reported in $12-52 \%$ of patients..$^{9,13,16}$ Similar to Garrhy et al. findings, symptomatic hypocalcemia in this study was also not significantly associated with hypomagnesemia. ${ }^{13}$ This was, however, in contrast to Wilson et al. finding, in 
which symptomatic hypocalcemia was associated with hypomagnesemia. ${ }^{12}$

Postoperative hypocalcemia and hypomagnesemia in this study were both significantly associated with preoperative hypocalcemia ( $p=0.03$ and $p=0.04$, respectively). This could suggest that patients with preoperative low levels of calcium may likely develop postoperative hypocalcemia. Hammerstad et al. has shown a significant association of preoperative hypocalcemia with permanent hypocalcemia. ${ }^{9}$ Other studies have shown significant association of postoperative hypomagnesemia with cancer diagnosis, central neck dissection and gender, ${ }^{11-13}$ and association of postoperative hypocalcemia with perioperative fluid use and low PTH postoperatively. ${ }^{11}$

One patient from present study developed persistent symptomatic hypocalcemia. Although she had normal magnesium and low calcium postoperatively, she developed severe and persistent signs and symptoms of hypocalcemia. Magnesium on the $9^{\text {th }}$ postoperative day was $1.6 \mathrm{mg} / \mathrm{dl}$ and calcium was $6.5 \mathrm{mg} / \mathrm{dl}$. This can suggest the importance of magnesium testing in severe signs or symptoms of hypocalcemia.

A drop in magnesium level was noted similar to other studies. ${ }^{9,13}$ In this study, the drop in magnesium after surgery was significantly greater in patients developing postoperative hypocalcemia. Hammerstad et al. previously showed a significant association between drop in magnesium level following surgery and permanent hypocalcemia. ${ }^{9}$ As no patient was hypomagnesemic preoperatively, a drop in magnesium may suggest a disturbance in the physiological relationship between magnesium and PTH, leading to hypocalcemia as Rude et al. suggested that hypomagnesemia may inhibit PTH secretion. ${ }^{22}$

One of the limitations of this study was the sampling and delivery of the blood samples to the laboratory facility. There was variation in timing for pre-op samples, as it depended on the timing of patients' admission prior to surgery and different members on duty for sampling. Unavoidable delays, due to logistic limitation, were also seen in some postoperative samples. Other limitations were not taken in account, like parathyroid gland resection peroperatively or PTH levels postoperatively, surgery by different surgeons, and a small sample size. However, the strengths of this study is that it excluded all other thyroidectemies other than total and near-total procedure. Preoperative sampling ensured a baseline level for all patients. No patient was started on prophylactic calcium in the first 24-hour postoperative period. To our knowledge, this was the first study in Pakistan to investigate magnesium levels in patients undergoing thyroidectomy. This study was able to quantitatively put the relationship between calcium and magnesium into an equation. This study may suggest that patients having low calcium levels preoperatively may likely develop postoperative hypocalcemia; therefore, it may be useful to further study the role of preoperative calcium levels in the development of postoperative hypocalcemia. While this study reinforces the biochemical relationship between calcium and magnesium, its clinical implication and therapeutic role must be further studied.

\section{CONCLUSION}

Postoperative hypomagnesemia was not a common finding in the study population. It was, however, significantly associated with postoperative hypocalcemia. Mean calcium levels were significantly lower in patients with hypomagnesemia; and there was a significant drop in magnesium levels in patients developing postoperative hypocalcemia. Monitoring of magnesium levels in patients developing severe or persistent hypocalcemia postoperatively, is recommended.

\section{ETHICAL APPROVAL:}

This observational cross-sectional study was conducted with the approval from the Ethical Review Committee of Rawalpindi Medical University, Rawalpindi.

\section{PATIENTS' CONSENT:}

Informed consents were taken from the patients, who were prepared for surgery as per the department's protocol.

\section{CONFLICT OF INTEREST:}

The authors declared no conflict of interest.

\section{AUTHORS' CONTRIBUTION:}

SV: Conception and planning of research, data acquisition, analysis, literature research, manuscript writing and review.

TA: Research planning, patient selection and sampling, data acquisition, manuscript review.

$\mathrm{NI}$ : Research planning, data acquisition, manuscript review. NZ: Conception and design, supervision of all stages of research, critical revision of data analysis and interpretation, manuscript review.

\section{REFERENCES}

1. Vetter T, Lohse MJ. Magnesium and the parathyroid. Curr Opin Nephrol Hypertens 2002; 11(4):403-10. doi:10.1097/ 00041552-200207000-00006.

2. Quitterer U, Hoffmann M, Freichel M, Lohse MJ. Paradoxical block of parathormone secretion is mediated by increased activity of G alpha subunits. J Biol Chem 2001; 276(9): 6763-9. doi:10.1074/jbc.M007727200.

3. Blaine J, Chonchol M, Levi M. Renal control of calcium, phosphate, and magnesium homeostasis. Clin J Am Soc Nephrol 2015; 10(7):1257-72. doi:10.2215/CJN.09750913.

4. Walker HK, Hall WD, Hurst JW, editors. Clinical Methods: The history, physical, and laboratory examinations. 3rd edition. Boston: Butterworths 1990; Chapter 143.

5. Fatemi S, Ryzen E, Flores J, Endres DB, Rude RK. Effect of experimental human magnesium depletion on parathyroid hormone secretion and 1, 25-dihydroxyvitamin D metabolism. J Clin Endocrinol Metab 1991; 73(5):1067-72. 
doi:10.1210/jcem-73-5-1067.

6. Pattou F, Combemale F, Fabre S, Carnaille B, Decoulx M, Wemeau JL. Hypocalcemia following thyroid surgery: Incidence and prediction of outcome. World J Surg 1998; 22(7):718-24. doi: 10.1007/s002689900459.

7. Page C, Strunski V. Parathyroid risk in total thyroidectomy for bilateral, benign, multinodular goitre: report of 351 surgical cases. J Laryngol Otol 2007; 21(3):237-41. doi:10.1017/ S0022215106003501.

8. Pfleiderer AG, Ahmad N, Draper MR, Vrotsou K, Smith WK. The timing of calcium measurements in helping to predict temporary and permanent hypocalcaemia in patients having completion and total thyroidectomies. Ann $R$ Coll Surg Engl 2009; 91(2):140-6. doi:10.1308/0035884 $09 \times 359349$.

9. Hammerstad SS, Norheim I, Paulsen T, Amlie LM, Eriksen EF. Excessive decrease in serum magnesium after total thyroidectomy for Graves' disease is related to development of permanent hypocalcemia. World J Surg 2013; 37(2):369-75. doi:10.1007/s00268-012-1843-2.

10. Jones CT, Sellwood RA, Evanson JM. Symptomatic hypomagnesaemia after parathyroidectomy. Br Med J 1973; 3(5876):391-2. doi:10.1136/bmj.3.5876.391.

11. Cherian AJ, Gowri M, Ramakant P, Paul TV, Abraham DT, Paul MJ. The Role of Magnesium in Post-thyroidectomy Hypocalcemia. World J Surg 2016; 40(4):881-8. doi:10.1007/ s00268-015-3347-3.

12. Wilson RB, Erskine C, Crowe PJ. Hypomagnesemia and hypocalcemia after thyroidectomy: prospective study. World J Surg. 2000; 24(6):722-6. doi:10.1007/s00 2689910116.

13. Garrahy A, Murphy MS, Sheahan P. Impact of postoperative magnesium levels on early hypocalcemia and permanent hypoparathyroidism after thyroidectomy. Head Neck 2016; 38(4):613-9. doi:10.1002/hed.23937.

14. Sousa Ade A, Salles JM, Soares JM, Moraes GM, Carvalho JR, Savassi-Rocha PR. Evolution of blood magnesium and phosphorus ion levels following thyroidectomy and correlation with total calcium values. Sao Paulo Med J 2010; 128(5):268-71. doi:10.1590/s1516-31802010000500005.

15. Wang X, Zhu J, Liu F, Gong Y, Li Z. Postoperative hypomagnesaemia is not associated with hypocalcemia in thyroid cancer patients undergoing total thyroidectomy plus central compartment neck dissection. Int J Surg 2017; 39:192-6. doi:10.1016/j.ijsu.2017.01.085.

16. Chincholikar SP, Ambiger S. Association of Hypomagnesemia with Hypocalcemia after Thyroi-dectomy. Indian J Endocrinol Metab 2018; 22(5):656-60. doi:10.4103/ijem.lJEM_599_17.

17. Umar M, Baloch N, Mehmood Z, Ali M, Ali A. Transient hypocalcaemia in total versus near total thyroidectomy. J Surg Pakistan 2017; 22(3):87-91. doi: dx.doi.org/ 10.21699/jsp.22.3.5.

18. Iqbal MA, Subhan AN, Baig MS, Shah MS. Frequency of hypocalcaemia in total thyroidectomy. J Surg Pak 2010; 15(2):87-91.

19. Saleem MA, Shaukat A, Masood S, Asif HM, Bukhari SH, Ali G. Frequency of post-operative hypocalcaemia with or without pre-operative calcium supplementation after total thyroidectomy in hyperthyroidism. Pak Postgrad Med J 2019; 30(1):36-42.

20. Ono T, Kitaguchi K, Takehara M, Shiiba M, Hayami K. Serum-constituents analyses: effect of duration and temperature of storage of clotted blood. Clin Chem 1981; 27(1):35-38.

21. Wang W, Meng C, Ouyang Q, Xie J, Li X. Magnesemia: An independent risk factor of hypocalcemia after thyroidectomy. Cancer Manag Res 2019; 11:8135-44. doi:10. 2147/CMAR.S218179.

22. Rude RK, Oldham SB, Sharp CF Jr, Singer FR. Parathyroid hormone secretion in magnesium deficiency. J Clin Endocrinol Metab 1978; 47(4):800-6. doi:10.1210/jcem47-4-800. 